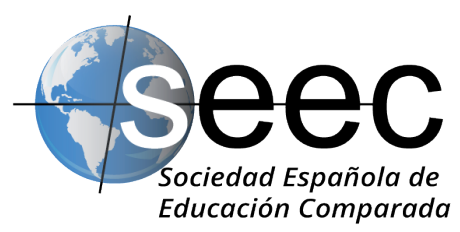

\title{
Las acreditaciones internacionales de los programas de ingeniería en tiempos de pandemia: perspectiva comparada entre España y México
}

International accreditations of engineering programs in times of pandemic: a comparative perspective between Spain and Mexico

\author{
Ana Isabel Bonilla-Calero*; \\ Enrique Morales-González ${ }^{* *}$; \\ María Ángeles Serrano-García ${ }^{* * *}$
}

DOI: $10.5944 /$ reec.40.2022.28373

Recibido: 14 de septiembre de 2021 Aceptado: 23 de noviembre de 2021

\footnotetext{
*Ana Isabel Bonilla-Calero: Jefa del Programa de Sellos Internacionales de Calidad de la Agencia Nacional de Evaluación de la Calidad y Acreditación (ANECA) y Directora de la Revista ACREDITAS por la Excelencia en Iberoamérica. Datos de contacto: E-mail:abonilla@aneca.es. ORCID: https://orcid.org/oooo-0003-2588-9769 **Enrique Morales-González: Colaborador de ANECA y académico de carrera a tiempo completo de la Universidad Veracruzana (México). Datos de contacto: E-mail:emorales@uv.mx

***María Ángeles SerRano-García: Directora de la División de Enseñanzas e Instituciones de ANECA y Catedrática de la Universidad de Salamanca (España). Datos de contacto: E-mail: mserrano@aneca.es
} 


\title{
Resumen
}

Introducción: En este trabajo se realiza una reflexión sobre las acreditaciones internacionales de programas educativos de ingeniería españoles y mexicanos, gestionadas por la Agencia Nacional de Evaluación de la Calidad y Acreditación (ANECA), en colaboración con el Instituto de Ingeniería de España (IIE) y el Consejo de Acreditación de la Enseñanza de Ingeniería (CACEI) de México, dentro del Programa de Sellos Internacionales de Calidad (SIC), desde sus inicios hasta el momento de la pandemia provocada por la COVID-19, en el contexto de la evaluación del Sello EUR-ACE® de ingeniería, creado por la agencia europea European Network for the Accreditation of Engineering Education (ENAEE). Materiales y Métodos: Los datos utilizados en este estudio fueron obtenidos directamente por ANECA, fruto de sus evaluaciones finales desde 2014 a julio 2020, de las que están en curso en ese mismo mes, junto con los más relevantes de los obtenidos en la metaevaluación anual del Programa SIC. Resultados y discusión: 234 programas educativos obtuvieron el Sello EUR-ACE@ en el periodo analizado, distribuidos entre 35 universidades españolas y tres mexicanas, en los que se aplicó idéntico proceso de evaluación y criterios. Predominaron los de las especialidades de ingeniería: «Información y Comunicación», «Industrial», «Química», «Civil/ Construcción» y «Mecánica», según la clasificación establecida por ENAEE. 197 correspondieron a Grados y 66 a programas de Máster. Debido a la pandemia provocada por la COVID-19, desde el 2020 la evaluación se está desarrollando de manera completamente virtual, mejora que se une a la que permitirá a los programas mexicanos, que hayan obtenido la acreditación con CACEI en un plazo inferior a dos años con resultado favorable en el momento de evaluación del Sello, someterse únicamente a la evaluación de los dos criterios específicos del Sello europeo. Este hecho ha conducido a un incremento en el número de solicitudes mexicanas recibidas en junio de 2020.

Palabras clave: Acreditaciones internacionales; Sello de Calidad; Acreditación enseñanzas, Ingeniería; Educación comparada; Pandemia.

\begin{abstract}
Introduction: This paper reflects on the international accreditations of Spanish and Mexican Engineering educational programs, managed by the National Agency for Quality Assessment and Accreditation (acronym in Spanish, ANECA), in collaboration with the Spanish Institute of Engineering and the Mexican Engineering Teaching Accreditation Council, within the International Quality labels Program (SIC) from its origin to the moment of the pandemic caused by COVID-19, in the context of the evaluation of the EUR-ACE $\AA$ Engineering label, created by the European Agency European Network for Accreditation of Engineering Education (ENAEE). Materials and Methods: The data used in this study were obtained directly from ANECA, as a result of its final evaluations from 2014 to July 2020, those ongoing in the same month and the potential ones to be carried out in 2021, together with the most relevant data obtained in the annual meta-evaluation of the SIC Programme. Results and discussion: 234 educational programs have obtained the EUR-ACE® label in the analysed period, distributed among 35 Spanish and three Mexican universities, in which the same evaluation process and criteria have been applied. "Information and Communication", "Industrial", "Chemical", "Civil / Construction" and "Mechanics" are the Engineering specialties, according to the classification established by ENAEE, with more labels. 197 are Bachelor level programs and 66 Master degree programs. Due to the pandemic caused by COVID-19, since 2020 the evaluation has been carried out completely virtual, an improvement that will allow Mexican programmes, which have obtained accreditation with CACEI in less than two years with a favourable result at the time of evaluation of the label, to submit only to the evaluation the two specific criteria of the European label. This has led to an increase in the number of Mexican applications received in June 2020.
\end{abstract}

Keywords: Egypt; Thanawiyya Amma Examination; Private Tutoring; Education Equity; Quality of Education. 


\section{Introducción}

El plan Bolonia ha favorecido la consolidación del Espacio Europeo de Educación Superior, (EEES) mejorando la movilidad, la confianza y la compatibilidad entre las instituciones de educación superior del continente europeo. Sin embargo, la carencia de iniciativa legislativa por parte de la Comunidad Europea, junto con la introducción de reformas de cada país de la UE, según la realidad del contexto de cada uno de ellos, y la autonomía propia de las universidades en cuestiones como la definición de los planes de estudio, hace que sigan existiendo aspectos y/o factores que deben ser analizados y mejorados hasta lograr una implantación plenamente satisfactoria (Colombo y Esteban, 2020), lo que ha provocado la alta demanda de sellos de asociaciones europeas, como European Network for the Accreditation of Engineering Education (ENAEE), que se fundó en febrero de 2006 y se implantó en el 2007, con los siguientes objetivos:

1) Crear confianza en los sistemas de acreditación de los programas de ingeniería en Europa, estableciendo unos objetivos de calidad comunes para los programas de ingeniería acreditados.

2) Desarrollar acuerdos voluntarios sobre la acreditación de los programas educativos de ingeniería y reconocer las cualificaciones de ingeniería.

3) Elaborar normas para los requisitos de competencia de los ingenieros/as graduados/as en Europa.

4) Facilitar el intercambio de información, que abarque todas las disciplinas de ingeniería y perfiles, cuyo reconocimiento internacional promueva la movilidad académica y profesional de forma sencilla.

5) Dar valor internacional y el reconocimiento de la cualificación de ingeniería mediante la concesión de un Sello a los programas, que cumplan una serie de estándares establecidos por académicos/as y profesionales de diferentes países.

Desde su creación, se han concedido más de 2.000 sellos en más de 29 países, algunos de ellos fuera de Europa, por ejemplo, México (ENAEE, 2021).

El centro de estudio de este artículo son los reconocimientos internacionales obtenidos por programas educativos españoles y mexicanos, en un proceso de evaluación gestionado por la Agencia Nacional de Evaluación de la Calidad y Acreditación (ANECA) desde el 2014 hasta el momento actual, en el contexto actual de una crisis sanitaria, a través de criterios establecidos por representantes del mundo académico y profesional de diferentes países a través de ENAEE. En estos reconocimientos se obtiene el Sello EUR-ACE® de ingeniería, concedido por dicha agencia a una institución de educación superior respecto a un programa formativo, que cumple una serie de estándares definidos de acuerdo con los principios de calidad, relevancia, transparencia, reconocimiento y movilidad contemplados en el Espacio Europeo de Educación Superior.

ANECA con este proyecto tiene el objetivo de ayudar a dar un paso más a las universidades, inmersas en un continuo proceso de transformación desde sus inicios y comprometidas con las necesidades y el permanente equilibrio entre cada contexto y tiempo, con la promoción de la calidad educativa y el ofrecimiento a las instituciones de educación superior españolas y mexicanas de un reconocimiento internacional en el ámbito de la ingeniería, en colaboración con el Instituto de Ingeniería de España (IIE), quien propone profesionales para participar en estas evaluaciones. 
Para gestionar la evaluación en México es preceptiva la firma de convenios de colaboración con las varias agencias de aseguramiento de calidad: el Consejo de Acreditación de la Enseñanza de Ingeniería (CACEI) para las evaluaciones de las licenciaturas y con los Comités Interinstitucionales para la Evaluación de la Educación Superior (CIEES), para las maestrías, quienes proponen evaluadores/as mexicanos/as, que pueden participar en evaluaciones de programas españoles y mexicanos, indistintamente. Ello es posible porque la formación es idéntica para todos/as, así como el proceso y criterios de evaluación utilizados en dichas acreditaciones españolas y mexicanas (ANECA, 2021a; Bonilla-Calero, 2019).

El principal objetivo de este estudio es compartir la experiencia de ANECA de estas acreditaciones internacionales en España y México, que aportan convergencia y visibilidad a los programas que las superan con resultados positivos, que se desglosa en los siguientes objetivos:

Presentar los resultados obtenidos en el período 2014-2020, distribuidos por país, universidad, ciclo formativo y especialidades de ingeniería establecidas por ENAEE.

Identificar las mejoras que se han implantado al Programa de Sellos Internacionales de Calidad, en el que se desarrollan estas evaluaciones internacionales desde su creación hasta la actualidad. Algunas surgidas para responder a la nueva situación derivada de la pandemia provocada por la COVID-19.

Mostrar los resultados de aprendizaje en los que se han identificado más dificultades para garantizar su integración y adquisición completa, a partir de los programas educativos evaluados hasta el momento, desde una perspectiva comparada entre España y México. Se aplica a todos los programas formativos, independientemente de su país de origen, el mismo procedimiento de evaluación y mismo nivel de exigencia en el cumplimiento de los criterios exigidos en estas acreditaciones para obtener el Sello EUR-ACE $\mathbb{R}$ de ingeniería.

Para 2025, el EEES diseñado desde la Comisión Europea considera que la educación y la cultura son los motores para generar empleo y crecimiento económico; el nuevo Espacio propone reforzar los principios y procesos identificados con el proyecto europeo (Ansejo y Ansejo, 2020) lo que, en tiempos de pandemia, hace que todos los/as representantes del mundo académico y profesional deban mostrar más unión y coherencia ante un futuro incierto.

La motivación que ha llevado a la publicación de este trabajo es el éxito obtenido en el desarrollo de las evaluaciones de la convocatoria 2019 y 2020 en cuanto a plazos y al interés por nuevas solicitudes para próximas convocatorias. La pandemia no ha paralizado la finalización de las evaluaciones de la convocatoria 2019, ni ha aplazado el inicio de las de la convocatoria 2020, que en ambos casos coincidió en marzo del 2020, un momento en el que se limitaban las actividades presenciales. ANECA ofreció a todas las universidades implicadas la posibilidad de aplazar estas evaluaciones ante la nueva situación derivada de la crisis sanitaria, teniendo en cuenta que estas acreditaciones internacionales son voluntarias, cuestan tiempo y dinero a las universidades evaluadas y que el proceso de evaluación tenía que adaptarse en pocas semanas a la «nueva normalidad» al pasar una parte de la evaluación (visita al centro y entrevistas) de la modalidad presencial a la virtual. Opción que no fue elegida por las universidad y tampoco en el sondeo realizado en junio de ese mismo año a todas las universidades, para conocer quiénes estarían interesadas en presentar programas a evaluar en la convocatoria 2021, porque universidades que estaban participando en las convocatorias 2019 y 2020 han manifestado su interés 
en el Programa SIC con nuevos programas, , incluso nuevas universidades españolas, mexicanas e de un país que hasta ahora no había solicitado evaluaciones, Ecuador.

Cada vez más instituciones de educación superior son conscientes de los beneficios que aporta la obtención de este Sello para ellas, para los/as egresados/as de los programas formativos con Sello y para las empresas que emplean a dichos/as egresados/as, tanto en España como en México, país que hasta ahora ha participado en varias convocatorias gestionadas por ANECA.

Si los centros formativos buscan una finalidad educativa próxima a las metas y valores del EEES, el registro de unos contenidos parcelados en áreas curriculares no puede estar más alejado de ello ya que la educación no es una acumulación de información, ni un mero proceso de capacitación. La educación es un elemento de cambio social, generador de cohesión social, impulsado por el proyecto de una Europa educada y educadora que está predispuesta a participar en acciones de movilidad para formar parte de la cultura del encuentro y la cohesión (Ansejo y Ansejo, 2020; Kant, 1999). De ahí, que ya sean muchas las universidades, que voluntariamente, presentan sus programas formativos españoles y mexicanos a un riguroso proceso de evaluación externa, en el que debe demostrar el cumplimiento de estándares definidos, de acuerdo con los principios de calidad, relevancia, transparencia, reconocimiento y movilidad contemplados en un contexto internacional, en el que se ha aprobado la impartición de estos programas.

\section{Materiales y métodos}

Los datos utilizados en este estudio han sido proporcionados por la Agencia Nacional de Evaluación de la Calidad y Acreditación (ANECA), encargada de gestionar estas acreditaciones internacionales, Agencia autorizada por la asociación internacional ENAEE para evaluar la obtención de este Sello EUR-ACE ${ }^{\circledR}$ de ingeniería, en colaboración con el IIE, una vez superado un proceso de evaluación que duró aproximadamente doce meses entre los años 2013-2014.

Los programas formativos españoles y mexicanos que solicitan la evaluación para la obtención de este Sello, deben someterse a un procedimiento de evaluación definido por personal de ANECA, en colaboración con expertos/as académicos y profesionales nacionales e internacionales, aprobado y auditado cada cinco años por ENAEE.

Un procedimiento que no puede superar doce meses contados desde el día que la institución de educación superior presenta el informe de autoevaluación y las evidencias exigidas para la evaluación (momento de iniciarse el procedimiento) hasta que ANECA emite la decisión final sobre la adjudicación del sello a través de una Comisión de Acreditación del Sello. A la mitad del proceso se realiza una visita virtual al centro en el que se imparte el programa evaluado por un panel de evaluación externa, que es el encargado de entrevistar a todos los agentes implicados en él: equipo directivo, profesorado, estudiantado, egresados/as y empresas que los/as contratan a estos/as últimos.

A partir de la información obtenida en el análisis del informe de autoevaluación y evidencias, que presenta la universidad, junto a la recopilada durante la visita, el panel de evaluación externa elabora un informe de evaluación. Este equipo está compuesto por: dos académicos/as, uno de ellos ejerce como presidente/a, un/a profesional, un/a estudiante y un/a tercer/a académico/a o técnico/a de calidad, que ejerce como secretario/a de dicho equipo, para garantizar que la evaluación se realiza cumpliendo la normativa establecida por ANECA para estas evaluaciones, que cada cinco años se somete a aprobación por ENQA y, como ya se ha citado previamente, también, por ENAEE. 
Este informe de evaluación se envía a la Comisión de Acreditación del Sello, encargada de emitir una evaluación provisional, primero, y final después, una vez analizadas las posibles alegaciones que pueda presentar cada universidad al informe derivado de la evaluación provisional. Esta Comisión está compuesta por: 6 académicos/as y 6 profesionales del ámbito de la ingeniería, uno/a de ellos/as ejerce de presidente. Esta Comisión, también, cuenta con un séptimo/a académico/a o técnico/a de calidad, que ejerce como secretario/a, que puede ser de un ámbito diferente al de ingeniería, al no tener un rol de evaluador/a. Él/ella es el/a encargado/a de garantizar que la evaluación cumpla toda la normativa establecida por ANECA, previamente al inicio de esta. Los/as evaluadores/as de esta Comisión cuentan con amplia experiencia internacional o su centro de trabajo está en un país diferente a España, en el momento de su participación en dicha comisión, debido a la importancia de la internacionalización de este Sello.

A partir del desarrollo de estas acreditaciones internacionales se obtienen los siguientes datos:

1) Los de evaluación:

$\sqrt{ } \quad$ obtenidos desde la convocatoria piloto (celebrada en el año 2014) a la convocatoria 2019 (finalizada en 2020): número de evaluaciones; resultados finales positivos versus negativos, distribución de evaluaciones positivas por país, universidad, ciclo y especialidad de ingeniería; debilidades identificadas en los programas educativos evaluados según los criterios de ENAEE.

$\sqrt{ } \quad$ los que están en curso en la convocatoria 2021 y los potenciales a presentarse a evaluar en el 2022: número de evaluaciones distribuidas por país y por número de universidades.

2) Los identificados en la metaevaluación realizada anualmente al Programa de Sellos Internacionales de Calidad (SIC), a partir del análisis de las encuestas de satisfacción cumplimentadas por todas las universidades españolas y mexicanas y evaluadores/as que participan cada año en cada convocatoria.

En el caso de las evaluaciones finalizadas, se establece la siguiente clasificación de posibles resultados:

a) Obtención del Sello: para aquellos programas educativos oficiales que hayan conseguido una valoración A (se supera excelentemente) o B (se alcanza) en todos los criterios de evaluación. La obtención del Sello podrá incluir, en cualquier caso, recomendaciones para la mejora del programa, y tendrá una validez de seis años en el caso de los títulos de Grado y de cuatro para el caso de los títulos de Máster.

b) Obtención del Sello con prescripciones: para aquellos programas que, habiendo conseguido valoraciones $\mathrm{A}$ o $\mathrm{B}$ en la mayor parte de los criterios de evaluación, cuenten con alguna valoración C (se alcanza parcialmente) en algún criterio.

Si el título obtiene el Sello con prescripciones, estas se especifican en cuanto a tiempo y forma en el informe de evaluación final emitido por ANECA, y su consecución debe ser posible dentro de un plazo de tiempo razonable (por regla general inferior a la mitad del periodo total habitual de la renovación de la acreditación). La Comisión de Acreditación 
del Sello es la encargada de determinar la fecha de verificación del cumplimiento de las prescripciones.

La adjudicación del Sello se hace en función de la aceptación de las prescripciones por parte de la universidad, incluidas en el informe final de evaluación para la obtención del Sello. Una vez cumplido ese periodo se comprueba el cumplimiento de las prescripciones señaladas en dicho informe. De no cumplirse las prescripciones establecidas en el informe final de evaluación para la obtención del Sello, este dejará de tener validez para ese título.

c) Denegación del Sello: para aquellos casos en los que el procedimiento de evaluación determine que no se alcanzan los criterios y estándares previamente establecidos en la evaluación del mismo.

Para el análisis de datos, se recomienda el uso de evaluaciones finalizadas y no de asignadas a convocatoria, dado que las evaluaciones pueden estar asignadas a una convocatoria y haber sido evaluadas en un año diferente a este. Desde el año 2018 lo habitual es iniciar la evaluación a finales del año de la convocatoria y finalizarlo en el siguiente, aunque desde ANECA se están haciendo ajustes en las planificaciones de las evaluaciones, para conseguir que las evaluaciones se finalicen el mismo año en el que se inician.

\section{Resultados y discusión}

\subsection{Las acreditaciones internacionales en el ámbito de ingeniería en España y México (2014-2020)}

Desde 2014 a 2020 se han realizado 263 evaluaciones. Están en activo 83 distribuidas entre 24 universidades, tres de ellas mexicanas, que entre todas alcanzan un total de 18 evaluaciones y se han recibido 146 solicitudes para presentarse a la convocatoria 2022 del Sello de ingeniería distribuidas entre: 19 universidades españolas, 5 mexicanas y 3 ecuatorianas (ANECA, 2021b).

Cada año entre junio y noviembre se ofrece un tiempo de reflexión a las universidades que han manifestado su interés en presentar programas a la evaluación del Sello europeo de ingeniería, para que realicen un análisis de las garantías de éxito con las que cuentan para conseguir dicho Sello, una vez que ANECA les facilita el procedimiento de evaluación y documentación que debe presentar cada universidad por cada programa educativo a evaluar y les ofrece la resolución de todas las dudas que les puedan surgir a los/as interesados/as al respecto.

Durante este análisis hay universidades que detectan que no cumplen todos los criterios para conseguir el Sello o que necesitan más tiempo para poderse presentar con evidencias suficientes y/o adecuadas para justificar que cumplen todos ellos. Por ejemplo, 40 solicitudes de la convocatoria 2021 fueron aplazadas para próximas convocatorias por este motivo.

Respecto a las evaluaciones solicitadas y ya realizadas, en la Tabla 1 se muestran los datos desde el año 2014, en el que hubo dos convocatorias: la piloto con 18 evaluaciones y la primera ordinaria, con 7 , hasta julio del 2020 en el que se ha finalizado la convocatoria 2019, iniciada en dicho año. Entre el inicio y cierre de la evaluación de un programa educativo pueden transcurrir hasta 12 meses. La universidad elige cuándo presenta la documentación a evaluar entre marzo y mayo del año de la convocatoria y desde esa 
fecha se establecen entre dos o tres meses para realizar la visita a la universidad, con el objetivo de entrevistar a los diferentes agentes implicados en el programa educativo a evaluar: equipo directivo, profesorado, estudiantado, egresados/as y empleadores/as.

Si se observa el número de evaluaciones finalizadas (263), existe un incremento desde el 2014 al 2020, de 20 evaluaciones al año en el 2014 a 60 en los seis primeros meses del 2020, sin verse afectado esta tendencia creciente por la crisis sanitaria provocada por la COVID-19, porque las universidades que se han presentado a la convocatoria 2019 y 2020 se han preocupado y esforzado, tanto en España como en México, por cumplir todos los plazos de evaluación, aportando en tiempo y forma toda la documentación solicitada por los/as evaluadores/as durante dicho proceso. En México las primeras 6 evaluaciones se finalizaron en el 2016 y le han seguido 4 más, distribuidas simétricamente entre la convocatoria 2019 y 2020. Al cierre de este artículo se están evaluando 18.

Tabla 1.

Evaluaciones asignadas a convocatoria versus evaluaciones finalizadas por año

\begin{tabular}{|c|c|c|c|c|}
\hline & & $\begin{array}{l}\text { Evaluaciones } \\
\text { finalizadas }\end{array}$ & $\begin{array}{c}\text { Evaluaciones } \\
\text { finalizadas }\end{array}$ & $\begin{array}{c}\text { Total } \\
\text { finalizadas }\end{array}$ \\
\hline Año & Evaluaciones asignadas & ESPAÑA & MÉXICO & \\
\hline Piloto (2014) & 13 & 13 & & 13 \\
\hline 2014 & 7 & 7 & & 7 \\
\hline 2015 & 88 & 23 & & 23 \\
\hline 2016 & 21 & 19 & 6 & 25 \\
\hline 2017 & 12 & 27 & & 27 \\
\hline 2018 & 62 & 54 & & 54 \\
\hline 2019 & 60 & 52 & 2 & 54 \\
\hline 2020 & $50^{*}$ & 58 & 2 & 60 \\
\hline Total & 320 & 253 & 10 & 263 \\
\hline
\end{tabular}

*Están en proceso de evaluación. Su finalización está prevista en el 2021.

En la Tabla 2 se observa que el porcentaje de obtención del Sello es muy alto (89 \%) respecto al total de evaluaciones realizadas hasta el momento. Dentro de los resultados favorables se establecen dos valoraciones: 1) obtención, por la que se concede el Sello hasta seis años, si es un Grado, y hasta cuatro, si es un Máster; 2) obtención con prescripciones, en aquellos programas en que se tienen que subsanar, por parte de la universidad, una serie de debilidades, identificadas por los/as evaluadores/as, en un periodo máximo de tres años para los Grados y de cuatro para los programas de Máster, con el objetivo de mantener el Sello un total de seis años o cuatro, dependiendo del ciclo del programa educativo evaluado. De los programas mexicanos hasta ahora solo uno de ellos ha sido valorado con el resultado «Obtención». 
Tabla 2.

Resultados finales positivos (obtención y obtención con prescripciones) versus negativos (denegación)

\begin{tabular}{lcc}
\hline \multicolumn{1}{c}{ Tipo de resultado } & $\begin{array}{c}\text { Número } \\
\text { evaluaciones }\end{array}$ & \% \\
\hline Obtención & $\mathbf{1 3 3}$ & $\mathbf{5 0 , 5 7}$ \\
\hline Obtención con prescripciones & $\mathbf{1 0 1}$ & $\mathbf{3 8 , 4 0}$ \\
\hline Denegación & 29 & $\mathbf{1 1 , 0 3}$ \\
\hline Total & $\mathbf{2 6 3}$ & \\
\hline
\end{tabular}

Es importante señalar que al tratarse de una evaluación voluntaria no todos los programas educativos de ingeniería, que podrían presentarse a evaluación, la solicitan. Incluso, algunos que durante la consulta, que se hace a todas las universidades en el mes de junio, para saber si presentarán en la próxima convocatoria, que manifiestan su interés en ese momento, cuando se abre la convocatoria oficial en el mes de noviembre, no confirman su participación en la siguiente convocatoria (una media de 30 solicitudes al año), para poder hacer cambios en sus planes de estudio y obtener nuevas evidencias que demuestren claramente el cumplimento de todos los criterios establecidos por ENAEE, con el objetivo de tener más garantía de éxito de conseguir el Sello en el futuro. Como ha pasado en la convocatoria 2021 con universidades españolas, mexicanas y chilenas.

Uno de los motivos principales de denegación, hasta el momento, es no demostrar en siete o más de los sub-resultados de aprendizaje establecidos por ENAEE para conseguir este Sello, su integración completa en el programa educativo evaluado y/o su adquisición total por todos/as los/as estudiantes, una vez finalizado. En los programas de Grado se establecen 22 sub-resultados y en los de Máster, 28 sub-resultados, en ambos casos agrupados en ocho bloques: Conocimiento y comprensión; Análisis en ingeniería; Proyectos de ingeniería; Investigación e innovación; Aplicación práctica de la ingeniería; Elaboración de juicios; Comunicación y trabajo en equipo y Formación continua.

Respecto a los motivos por los que se han emitido 101 informes de evaluación con el resultado de «obtención con prescripciones» durante el periodo analizado (Tabla 3), entre los que suman 224 prescripciones, es necesario mencionar que cada informe puede contener entre 1 a 6 prescripciones, relativas a los sub-resultados de aprendizaje establecidos por ENAEE, agrupados en ocho bloques, porque su integración no es completa en el programa educativo evaluado y/o su adquisición no es total por todos los/as estudiantes, una vez cursado el programa educativo. En estos casos la Comisión de Acreditación del Sello establece un período entre dos a tres años, dependido el ciclo del programa evaluado, para subsanar estas debilidades, con el objetivo de conseguir una integración y adquisición de estos sub-resultados de forma completa, tanto en el programa educativo como por los/as estudiantes que lo cursan, respectivamente.

$\mathrm{Al}$ analizar en qué bloques de sub-resultados se han identificado más debilidades, «Proyectos de Ingeniería», «Aplicación práctica de la ingeniería» y «Comunicación y trabajo en equipo» son los que han alcanzado los datos más altos, tanto en los programas españoles como mexicanos. Los dos primeros por su componente práctico, que requiere de una importante dedicación por parte del estudiantado, que no se ha demostrado en los programas educativos con informes con prescripciones en este bloque de sub-resultados, y el tercero, por la necesidad de incrementar el uso de un segundo idioma en las actividades desarrollas en los programas educativos con prescripciones en este aspecto. La suma de las prescripciones de estos tres bloques representa el $60 \%$ del total. 
No se observan diferencias significativas entre los programas de Grado y Máster evaluados, ni entre los programas españoles y mexicanos en cuanto a las prescripciones emitidas por bloque de sub-resultados de aprendizaje.

Tabla 3.

Bloques de sub-resultados de aprendizaje en los que se han emitido prescripciones

\begin{tabular}{|c|c|c|c|c|}
\hline Resultados de aprendizaje & $\begin{array}{c}\text { No }^{\circ} \\
\text { prescripciones } \\
\text { ESPAÑA }\end{array}$ & $\begin{array}{c}\text { No } \\
\text { prescripciones } \\
\text { MÉXICO }\end{array}$ & $\begin{array}{c}\text { No } \\
\text { prescripciones } \\
\text { totales }\end{array}$ & $\begin{array}{c}\% \\
\text { total de } \\
\text { ambos } \\
\text { países }\end{array}$ \\
\hline Conocimiento y comprensión & 11 & 2 & 13 & 5,80 \\
\hline Análisis en ingeniería & 12 & 2 & 14 & 6,25 \\
\hline Proyectos de ingeniería & 44 & 8 & 52 & $\mathbf{2 3}, \mathbf{2 1}$ \\
\hline Investigación e innovación & 28 & 4 & 32 & 14,29 \\
\hline $\begin{array}{l}\text { Aplicación práctica de la } \\
\text { ingeniería }\end{array}$ & 39 & 6 & 45 & 20,09 \\
\hline Elaboración de juicios & 23 & 2 & 25 & 11,16 \\
\hline Comunicación y trabajo en equipo & 32 & 5 & 37 & 16,52 \\
\hline Formación continua & 6 & O & 6 & 2,68 \\
\hline Total & 195 & 29 & 224 & \\
\hline
\end{tabular}

Si se analizan los resultados por ciclo formativo (véase Tabla 4), la distribución es similar, tanto en las evaluaciones de Grado como de Máster. El resultado «obtención» es de entorno al 50 \%: en los Grados de un 49 \% y en los programas de Máster de un $55 \%$. El resto de evaluaciones en ambos casos, se distribuyen entre los resultados «obtención con prescripciones» $\mathrm{y}$ «denegaciones», teniendo las evaluaciones negativas los datos más bajos, $10 \%$ en Grados y del $14 \%$ en Máster.

En el caso de México, hasta el momento, todas las evaluaciones han sido de nivel de Licenciatura o Ingeniería, aunque varios representantes/as de Maestrías de ingeniería han contactado con ANECA para interesarse en estas acreditaciones internacionales.

Tabla 4.

Resultados de evaluaciones finales (Grado versus Máster)

\begin{tabular}{lccccccc}
\hline $\begin{array}{c}\text { Tipo de } \\
\text { resultado }\end{array}$ & Obtención & \% & $\begin{array}{c}\text { Obtención } \\
\text { prescripciones }\end{array}$ & \% & Denegación & \% & Total \\
\hline Grado & $\mathbf{9 7}$ & $\mathbf{4 9 , 2 4}$ & 80 & 40,61 & $\mathbf{2 0}$ & $\mathbf{1 0 , 1 5}$ & $\mathbf{1 9 7}$ \\
\hline Máster & $\mathbf{3 6}$ & $\mathbf{5 4 , 5 5}$ & 21 & 31,82 & $\mathbf{9}$ & $\mathbf{1 3 , 6 4}$ & $\mathbf{6 6}$ \\
\hline
\end{tabular}


Este Sello de ingeniería abarca las 21 especialidades (Véase Tabla 5), establecidas por ENAEE en su base de datos de programas de ingeniería de todos los países: https:// eurace.enaee.eu/node/163, entre los que se encuentran programas españoles y mexicanos, siendo «Información y Comunicación» en la que se concentran más programas, seguida por «Industrial», «Química», «Civil/Construcción» y «Mecánica». Entre todas suman el $57 \%$ de las evaluaciones finales positivas en el periodo analizado (2014-2020).

Tabla 5 .

Resultados finales positivos distribuidos por especialidad de ingeniería de los programas educativos evaluados

\begin{tabular}{|c|c|c|}
\hline Especialidad & $\begin{array}{c}\text { Número de evaluaciones } \\
\text { positivas }\end{array}$ & $\%$ \\
\hline Información y comunicación & 49 & 20,94 \\
\hline Industrial & 25 & 10,68 \\
\hline Química & 21 & 8,97 \\
\hline Civil / Construcción & 19 & 8,12 \\
\hline Mecánica & 19 & 8,12 \\
\hline Agrícola / Alimentario & 15 & 6,41 \\
\hline Eléctrica y Electrónica & 13 & 5,56 \\
\hline Automatización & 12 & 5,13 \\
\hline Ambiental / Forestal & 10 & 4,27 \\
\hline Producción manufacturera & 9 & 3,85 \\
\hline Aeronáutica / Aeroespacial & 7 & 2,99 \\
\hline Electrónica & 7 & 2,99 \\
\hline Energía & 5 & 2,14 \\
\hline Ciencias de la Computación & 5 & 2,14 \\
\hline Bio/Biomédico & 5 & 2,14 \\
\hline Geológico / Geofísico / Geométrico & 4 & 1,71 \\
\hline Materiales / Textiles / Cerámica & 3 & 1,28 \\
\hline Automoción / Transporte & 2 & 0,85 \\
\hline Transporte & 2 & 0,85 \\
\hline Marina & 1 & 0,43 \\
\hline Naval & 1 & 0,43 \\
\hline Total & 234 & \\
\hline
\end{tabular}

Respecto a las 29 evaluaciones con resultado denegado, no se observa una concentración en ninguna de las especialidades de ingeniería, habiendo denegaciones en varias con datos similares.

En la Tabla 6 se muestra el número de acreditaciones internacionales distribuidas por 38 instituciones de educación superior (ANECA, 2021b), tres de ellas mexicanas (ubicadas al final de la Tabla). Entre las españolas destacan las universidades politécnicas, la Politécnica de Madrid y la Politècnica de València, ambas con 30 sellos obtenidos en el periodo analizado. En el caso de México, la universidad con más sellos en el periodo analizado, es la Universidad Autónoma de Nuevo León con cuatro sellos. 
Tabla 6.

Resultados de acreditaciones internacionales distribuidas por instituciones de educación españolas y mexicanas

\begin{tabular}{|c|c|c|}
\hline País & Instituciones de educación superior & Número de sellos \\
\hline España & Universidad Politécnica de Madrid & 30 \\
\hline España & Universitat Politècnica de València & $\mathbf{3 0}^{*}$ \\
\hline España & Universidad de Sevilla & 14 \\
\hline España & Universidad Carlos III de Madrid & 13 \\
\hline España & Universidad de Navarra & 12 \\
\hline España & Universidad de Málaga & 11 \\
\hline España & Universidad Pública de Navarra & 8 \\
\hline España & Universidad de Extremadura & 7 \\
\hline España & Universidad de Jaén & 7 \\
\hline España & Universidad de Alcalá & 6 \\
\hline España & Universitat D’Alacant & 6 \\
\hline España & Universidad Miguel Hernández & 5 \\
\hline España & Universitat Jaume I de Castellón & 5 \\
\hline España & Universitat Rovira i Virgili & 5 \\
\hline España & Universidad de Granada & 5 \\
\hline España & Universidad de Vigo & 5 \\
\hline España & Universidad Santiago de Compostela & 5 \\
\hline España & Universidad de Valladolid & 5 \\
\hline España & Universitat de València & 4 \\
\hline España & Mondragon Unibersitatea & 4 \\
\hline España & Universidad Politécnica de Cartagena & 4 \\
\hline España & Universidad Pontificia de Comillas & $4^{*}$ \\
\hline España & Universidad Rey Juan Carlos & 4 \\
\hline España & Universidad de Castilla-La Mancha & 4 \\
\hline España & Universidad de Córdoba & 4 \\
\hline España & Universidade da Coruña & 3 \\
\hline España & Universidad de Cantabria & 3 \\
\hline España & Universidad de la Laguna & 3 \\
\hline España & Universitat de les Illes Balears & 2 \\
\hline España & Universidad de País Vasco & 2 \\
\hline España & Universidad de Zaragoza & 2 \\
\hline España & Universidad de Oviedo & 1 \\
\hline España & Universidad Autónoma de Madrid & 1 \\
\hline España & Universidad de Almería & 1 \\
\hline España & Universidad Complutense & 1 \\
\hline México & Universidad Autónoma de Nuevo León & 4 \\
\hline México & Universidad Autónoma de Yucatán & 2 \\
\hline México & Universidad Autónoma San Luis de Potosí & 2 \\
\hline Total & & 234 \\
\hline
\end{tabular}

*Han renovado el sello en algunos de sus programas. La Universidad Politécnica de Valencia en dos y la Universidad Pontifica de Comillas en uno. 


\subsection{Las mejoras implantadas en el Programa SIC de acreditaciones internacionales en España y México}

Desde la convocatoria 2018, todos los años se realiza una metaevaluación del Programa SIC en la que se analizan las oportunidades de mejora identificadas en cada convocatoria finalizada, sin hacer distinción si se ha detectado en una evaluación en España o en México, a partir de:

1) las encuestas de satisfacción cumplimentadas por las universidades y evaluadores/as que participan en cada convocatoria, tanto en España como en México, enviadas a ANECA tras la finalización de cada evaluación,

2) las reuniones y correos recibidos sobre el programa SIC (11.127 en el año 2018 y 13.938 en el 2019) desde universidades españolas y mexicanas y evaluadores/ as de diferentes países por la perspectiva global de estas acreditaciones,

3) la reflexión realizada en ANECA, cuando se preparan las autoevaluaciones de SIC, previamente a las evaluaciones de este programa por ENAEE, por European Association for Quality Assurance in Higher Education (ENQA) y por European Quality Assurance Register for Higher Education (EQAR) cada cinco años,

4) el análisis y ajuste del procedimiento y criterios de evaluación SIC al incorporar nuevos sellos a dicho programa. En este momento, se gestionan dos sellos más: uno de informática y otro de química; y se ha implantado en el 2021 un nuevo Sello de Enseñanza No Presencial e Híbrida y otro de Medicina. Todos aplicables a programas españoles y mexicanos.

Todas las oportunidades de mejora que se identifican cada año se presentan ante la Comisión Técnica del Programa SIC, el comité encargado de velar por el buen funcionamiento del procedimiento de evaluación de Sellos, así como de la revisión de toda la documentación utilizada en él. Esta Comisión está compuesta por la Directora de la División Evaluación de Enseñanzas e Instituciones, la Jefa del Programa SIC, adscrita a dicha División, representantes del profesorado elegidos/as por ANECA y profesionales propuestos/as por IIE, la asociación con la que colabora ANECA en el Programa SIC. Todos con experiencia en procesos de calidad universitaria en España y México.

Se han implementado 199 oportunidades de mejora entre las convocatorias 20182020, de las que el 50 \% han sido identificadas por el equipo SIC. El 50 \% restante por los otros agentes implicados en este proyecto internacional: universidades, evaluadores/ as, asociaciones colaboradoras.

A continuación, se muestran las que afectan a las universidades, tanto españolas como mexicanas:

1) una nueva estructura de presentación de las evidencias, que justifican la integración de los sub-resultados de aprendizaje establecidos por ENAEE en el plan de estudios evaluado. Se ha pasado del formato en Word al formato en Excel. Se ha unificado la información de varias tablas y se aplicado el uso de filtros para su búsqueda.

2) las escalas «A/B/C/D/No Aplica» se han eliminado del informe de autoevaluación, que se ha convertido en una herramienta de información complementaria y aclaratoria a las evidencias presentadas por las universidades, que son 
la documentación clave para justificar el cumplimiento de cada directriz. En las plantillas de los informes de auto evaluación se ha incorporado la información mínima en la que se basa el análisis (evidencias e indicadores) y aspectos a valorar para el cumplimiento de las directrices de cada uno de los criterios, que se incluye en la guía de evaluación, de tal forma que la universidad solo tenga que utilizar un único documento durante la preparación de la documentación a evaluar.

3) la presentación de evidencias se ha convertido en un proceso completamente electrónico, mediante la creación de un repositorio documental y un manual de uso de este. En los inicios del programa las segundas evidencias, por ejemplo, dosieres de asignaturas de referencia, se facilitaban en papel durante la visita de los/as evaluadores/as a la universidad.

4) una nueva plantilla orientativa para utilizar en la presentación de los CVs de los/as profesores/as. No obstante, si una universidad ya cuenta con un formato de CV de sus profesores/as diferente, pero que atiende en contenido a lo exigido en estas evaluaciones, no es necesaria su adaptación a este y nueva plantilla para presentación de alegaciones, hasta este momento no existía, dado que el formato de envío de las alegaciones era libre.

5) la asignación en créditos a cada sub-resultado, ha sido eliminada, ya que se ha decidido que lo más importante en este apartado es mostrar claramente con qué profesorado, contenidos, actividades formativas y sistemas de evaluación se demuestra que todo el estudiantado trabaja de forma completa todos los subresultados establecidos por cada agencia internacional y con qué evidencias (trabajos y exámenes corregidos) se garantizan las adquisición de estos sub-resultados de aprendizaje por todos los/as egresados/as.

6) las categorías «básico», «adecuado» y «avanzado» de nivel de integración de los sub-resultados, se han eliminado en la convocatoria 2020, tras observarse en la metaevaluación del año 2019 que no facilitaban la evaluación, al no realizarse una asignación que demostraba claramente la categoría asignada en todos los casos, dado que el principal objetivo de esta evaluación es justificar la elección de las asignaturas con las que se verifica la integración de cada sub-resultado de aprendizaje, mediante el resumen de contenidos, actividades formativas y sistemas de evaluación, que se aportan para cada una de ellas, junto al CV del profesorado que las imparten y sus guías docentes y resultados obtenidos en ellas.

7) el nombre o código identificativo de los/as estudiantes de las asignaturas de referencia se presenta por la universidad al panel de expertos/s a partir de la convocatoria 2020, previamente a la selección de los dosieres de cada una de las asignaturas de referencia, con el objetivo de conseguir una selección menos arbitraria por parte del panel de expertos/as de dichos dosieres y de las personas que son entrevistadas durante la visita a la universidad.

8) un documento de Preguntas Frecuentes y un Glosario que se publican en la página web de ANECA y que se actualizan cada año, previamente a la apertura de cada convocatoria.

9) los informes de evaluación, que aportan una información más detallada, han aumentado su extensión de 4 hasta 40 páginas, para poder hacer alusión a como 
se integra y se adquiere cada sub-resultado de aprendizaje, de tal forma que la universidad pueda identificar más fácilmente, en los casos en los que el resultado no es «obtención sin prescripciones», qué aspectos tiene que mejorar para cumplir las prescripciones establecidas en los informes finales, o qué debilidades han sido los motivos de la denegación del Sello.

10) los informes finales de evaluación incorporan, desde la convocatoria 2019, la fecha desde la que los egresados/as se beneficiarán de la obtención del Sello y se ha incluido un apartado para hacer alusión a las evidencias que se presentan en la fase de alegaciones, dado que en dicha convocatoria en 7 evaluaciones se ha modificado el resultado entre el informe provisional y el final, tras la evaluación de las alegaciones presentadas por las universidades a dichos informes provisionales.

11) en el informe de cumplimento de prescripciones se ha incluido una sección para hacer referencia a las recomendaciones emitidas en los informes finales.

12) un plan de ahorro para reducir costes en las tareas derivadas de las acreditaciones internacionales.

Dentro de este apartado, se incluye un análisis del número de créditos asignados a la integración y adquisición de cada uno de los resultados de aprendizaje establecidos por ENAEE, a partir de los programas evaluados en la convocatoria 2018 en los que se pedía a las universidades españolas y mexicanas esta información, porque se identificó como una oportunidad de mejora propuesta por las universidades de ambos países en la última meta evaluación. No obstante, hay que ser cuidadosos en la lectura de estos datos y no sacar conclusiones rápidas, porque la muestra utilizada no es significativa, y dada la escasez de los datos no se diferencia si esta asignación de créditos ha derivado en resultado de «obtención» o de «obtención con prescripciones».

Únicamente, el estudio se ha limitado a los resultados positivos obtenidos durante el periodo analizado, sin diferenciar entre «obtenciones» $\mathrm{u}$ «obtenciones con prescripciones», y a los créditos que se han identificado como efectivos para garantizar la adquisición de todos los sub-resultados de aprendizaje establecidos por ENAEE por todo el estudiantado o hasta en seis sub-resultados de forma parcial, una vez finalizada la evaluación, ya que en la fase inicial de las evaluaciones hay universidades que han asignado todos los créditos del plan de estudios a todos los resultados de aprendizaje de forma errónea y en otros casos, los/as evaluadores/as han observado que las universidades españolas y mexicanas tendrían que haber seleccionado otros créditos de sus planes de estudios en el momento de presentar la documentación a evaluar. Tras la nueva información proporcionada por las universidades, se han confirmado los créditos efectivos que han permitido conseguir los resultados positivos emitidos en los informes finales analizados.

En las Tablas 7 y 8 se muestra el número mínimo de créditos y máximo que se han asignado a los sub-resultados de aprendizaje, agrupados por ocho bloques según ENAEE: Conocimiento y Comprensión, Análisis en ingeniería, Proyectos de ingeniería, Investigación e innovación, Aplicación práctica de la ingeniería, Elaboración de juicios, Comunicación y trabajo en equipo y Formación continua. En la primera Tabla 7 se analizan los programas de Grado, entre los que se encuentran los programas educativos mexicanos, y en la Tabla 8, los de Máster. 
En la Tabla 7, el valor medio mínimo de asignación de créditos por todos los sub-resultados de aprendizaje en cada bloque es de 4, es decir, durante unas 120 horas, que se trabajan dentro de una o distribuidas entre varias asignaturas. El valor más alto se ubica en el bloque de sub-resultados de aprendizaje «Conocimiento y comprensión» con 23 créditos. Los siguientes bloques con mayor número de créditos asignados son los de perfil más práctico, como «Análisis en ingeniería», «Proyectos de ingeniería «y «Aplicación práctica de la ingeniería», con 16, 14 y 11 créditos respectivamente.

El número mínimo de créditos asignado por las universidades en los 35 informes analizados es de un crédito en al menos uno de los sub-resultados de cada bloque, como por ejemplo, el sub-resultado «Ser conscientes del contexto multidisciplinar de la ingeniería» del bloque «Conocimiento y Comprensión» en el que, también, se encuentra el sub-resultado con el valor más alto (80) en el sub-resultado «Conocimiento y comprensión de las matemáticas y otras ciencias básicas inherentes a su especialidad de ingeniería, en un nivel que permita adquirir el resto de las competencias del título». El reparto no puede ser similar entre los diferentes sub-resultados, porque dependiendo de la dificultad de adquisición de cada uno ellos, en unos el/a estudiante necesitará más horas de trabajo para consolidarlo frente a otros.

Tabla 7.

Número de créditos asignados a bloques de resultados de aprendizaje ENAEE (programas de Grado-convocatoria 2018)

\begin{tabular}{|c|c|c|c|c|}
\hline Resultados de aprendizaje & $\begin{array}{l}\text { No de sub- } \\
\text { resultados }\end{array}$ & $\begin{array}{c}\text { Número } \\
\text { medio } \\
\text { todos } \\
\text { sub- } \\
\text { resultados } \\
\text { aprendizaje }\end{array}$ & $\begin{array}{l}\text { Mínimo } \\
\text { créditos } \\
\text { asignados }\end{array}$ & $\begin{array}{c}\text { Máximo } \\
\text { créditos } \\
\text { asignados }\end{array}$ \\
\hline Conocimiento y comprensión & 3 & 23 & 1 & 80 \\
\hline Análisis en ingeniería & 2 & 16 & $\mathbf{1}$ & 39 \\
\hline Proyectos de ingeniería & 2 & 14 & $\mathbf{1}$ & 29 \\
\hline Investigación e innovación & 3 & 5 & $\mathbf{1}$ & 18 \\
\hline Aplicación práctica de la ingeniería & 6 & 11 & $\mathbf{1}$ & 23 \\
\hline Elaboración de juicios & 2 & 4 & $\mathbf{1}$ & 10 \\
\hline Comunicación y trabajo en equipo & 2 & 9 & 1 & 20 \\
\hline Formación continua & 8 & 6 & $\mathbf{1}$ & 21 \\
\hline
\end{tabular}

En el caso de los programas de Máster analizados (Tabla 8), el número mínimo medio de créditos asignados es 3, es decir, un total de unas 90 horas de dedicación. Todos ellos con perfil de ingreso que garantiza la adquisición de todos los resultados de aprendizaje establecidos por ENAEE para conseguir este sello de ingeniería. El número máximo medio es de 3,5 créditos, localizado en «Proyectos de ingeniería», uno de los bloques de sub-resultados de aprendizaje con mayor componente práctico. En el resto de resultados de aprendizaje se observa una asignación homogénea entre 3 a 4 créditos.

El número mínimo de créditos asignado por las universidades en los 11 informes analizados es de un crédito en al menos uno de los sub-resultados de cada bloque y el número máximo $(8,5)$ se ubica en el sub-resultado «Conocimiento con sentido crítico del amplio contexto multidisciplinar de la ingeniería y de la interrelación que existe entre los conocimientos de los distintos campos». El reparto entre los sub-resultados no puede ser 
homogéneo entre los diferentes sub-resultados, porque como en los Grados, la dificultad de adquisición de cada uno ellos es diferente, en unos se requiere más dedicación que en otros.

Tabla 8.

Número de créditos asignados a bloques de resultados de aprendizaje ENAEE (programas de Máster-convocatoria 2018)

\begin{tabular}{lcccc}
\hline \multicolumn{1}{c}{ Resultados de aprendizaje } & $\begin{array}{c}\text { Número medio } \\
\text { Nub-resul- } \\
\text { tados }\end{array}$ & $\begin{array}{c}\text { todos } \\
\text { sub-resultados } \\
\text { aprendizaje }\end{array}$ & $\begin{array}{c}\text { Mínimo } \\
\text { créditos } \\
\text { asignados }\end{array}$ & $\begin{array}{c}\text { Máximo } \\
\text { créditos } \\
\text { asignados }\end{array}$ \\
\hline Conocimiento y Comprensión & 4 & $\mathbf{3}$ & $\mathbf{1}$ & $\mathbf{8 , 5}$ \\
\hline Análisis en ingeniería & 4 & 3,2 & $\mathbf{1}$ & 3 \\
\hline Proyectos de ingeniería & $\mathbf{2}$ & $\mathbf{4 , 3}$ & $\mathbf{1 , 3}$ & 6,7 \\
\hline Investigación e innovación & 5 & $\mathbf{3}$ & $\mathbf{1 , 7}$ & 7,5 \\
\hline Aplicación práctica de la ingeniería & 6 & $\mathbf{3}$ & $\mathbf{1}$ & 6,9 \\
\hline Elaboración de juicios & $\mathbf{2}$ & $\mathbf{3}$ & $\mathbf{1}$ & 4,1 \\
\hline Comunicación y Trabajo en Equipo & $\mathbf{2}$ & 4 & $\mathbf{1}$ & 6,4 \\
\hline Formación continua & $\mathbf{2}$ & 3,6 & $\mathbf{1}$ & 7,3 \\
\hline
\end{tabular}

Se insiste en el escaso número de programas evaluados analizados para obtener estos datos y en no olvidar que no nos encontramos frente a una evaluación cuantitativa, sino más bien cualitativa, porque se pueden asociar más de 80 créditos a cada sub-resultado y no obtener el Sello, porque las actividades que están detrás de esas horas de trabajo no son del nivel ni del tipo adecuado para garantizar que todos/as los/as estudiantes trabajan cada sub-resultado o que los sistemas de evaluación asociados a dichas actividades no permiten comprobar que todo el estudiantado adquiere dichos sub-resultados.

Por ello, en el futuro, este será el centro del análisis, identificar buenas prácticas sobre el tipo de actividades formativas y sistemas de evaluación que permiten conseguir resultados positivos en el proceso de enseñanza-aprendizaje establecido por ENAEE. De ahí, que en la metaevaluación del año 2019 se eliminó la necesidad de indicar el número de créditos en los que se trabaja cada sub-resultado de aprendizaje dentro de los ocho bloques de resultados de aprendizaje, citados anteriormente.

Sin haber sido realizada en marzo del 2020 la meta evaluación anual respecto a la convocatoria de dicho año, que se realiza durante los meses de septiembre y octubre de cada año, se identificaron dos nuevas oportunidades de mejora:

13) realizar la evaluación de manera completamente virtual, ante la imposibilidad de desarrollar presencialmente las visitas como habitualmente se realizaban, porque en el momento de su celebración, entre junio y octubre de 2020, no se tuviera garantía de contar con las medidas de seguridad necesarias para proteger la salud de los/as evaluadores/as y representantes de las universidades durante las evaluaciones, debido a crisis sanitaria provocada por la COVID-19. Para poder implantar esta mejora, se envió a ENAEE una propuesta de evaluación completamente virtual para su aprobación, avalada por un plan de contingencia redactado a partir de los resultados obtenidos en la encuesta remitida a los/as 
160 evaluadores/as, que estaban colaborando en el Programa SIC de ANECA en el 2020, para conocer su opinión sobre la posibilidad de una visita virtual, sobre las posibles incidencias que podrían producirse, así como las soluciones a estas y las recomendaciones a tener en cuenta en su desarrollo. Se generó un decálogo para evaluadores/as y universidades con el objetivo de facilitar el desarrollo de las visitas bajo la modalidad virtual, una jornada de formación específica sobre visitas virtuales para los/as 160 evaluadores/as y un calendario adicional al de las reuniones informativo-técnicas, ya fijado, con las universidades españolas y mexicanas que participaban en la convocatoria 2020, a las que se les ofrecía un canal complementario al correo y teléfono para resolver todas las dudas relativas a esta nueva modalidad de visitas. $\mathrm{Al}$ cierre de este artículo ya se han realizado visitas virtuales en 24 universidades, todas ellas con una valoración positiva ante este cambio (Bonilla-Calero y Serrano-García, 2021).

14) una específica para el proceso de evaluación de los programas mexicanos, no volver a evaluar los criterios 1 a 7 contenidos en el modelo de evaluación establecido para la obtención de este Sello, si previamente el programa educativo ha sido evaluado por el Consejo de Acreditación de la Enseñanza de la Ingeniería (CACEI) en un periodo inferior a dos años, tras realizar una comparativa entre los criterios que debe cumplir un programa para obtener la acreditación CACEI versus los siete primeros criterios del modelo de evaluación del presente Sello por ANECA, a partir de la consulta realizada a evaluadores/as que participan en ambos procesos de evaluación. La acreditación de CACEI está basada en estándares internacionales, como los que rigen el Washington Accord, establecido en 1989, un acuerdo internacional entre organismos responsables de la acreditación de programas de ingeniería que reconoce la equivalencia substancial de los programas acreditados por esos organismos y recomienda que los/as egresados/as de programas acreditados por cualquiera de los signatarios sean reconocidos por los demás organismos de que cumplen los requerimientos académicos para iniciar la práctica de la ingeniería.

- El modelo de evaluación del presente Sello está compuesto por nueve criterios, el nueve «soporte institucional de la universidad al programa evaluado» y el más importante, el criterio 8 «Resultados de aprendizaje del Sello», por ser el que incluye las directrices respecto a la integración de los resultados de aprendizaje de ENAEE en el programa evaluado y la adquisición de todos estos por todos/as sus estudiantes, una vez finalizado, que deben cumplir los programas educativos para obtener dicho sello.

Estas dos últimas mejoras, tanto la virtualización total del proceso, como la evaluación solo de los criterios específicos europeos de este, han producido un aumento de solicitudes mexicanas en ANECA. Se ha pasado de un máximo de seis potenciales evaluaciones al año a las 32 solicitudes recibidas en el sondeo realizado en junio del 2021. Todo ello, porque estos cambios les permiten a las instituciones mexicanas ahorrar costes y tiempo en las evaluaciones, respectivamente, al disminuir los gastos derivados de los viajes de los/as evaluadores/as y al establecer un modelo de evaluación centrado en los requisitos específicos europeos. En dicho modelo no se va a volver a evaluar lo ya evaluado por una agencia de aseguramiento de calidad mexicana, con la que se haya firmado un convenio 
de colaboración y tras realizar un análisis comparativo de criterios, que demuestre que se cubren todos los requisitos que un programa educativo debe cumplir para conseguir este Sello.

Esta mejora se podrá extender a otros países con los que ANECA tenga convenios de colaboración con su/s agencia/s de aseguramiento de calidad, como el caso de Ecuador, del que, también, se han recibido 18 solicitudes en el sondeo realizado en junio de 2021.

\section{Conclusiones}

- Con el objetivo de dar un paso más en promover la calidad educativa superior y el reconocimiento internacional de los/as egresados/as en el ámbito de ingeniería se implanta el Sello Internacional Calidad EUR-ACE®, creado por ENAEE, entre las actividades de evaluación de ANECA, que firma convenios de colaboración con: IIE, CACEI y CIEES. Con el IIE con el objetivo de incluir en los equipos de evaluación de ANECA la participación de profesionales de ingeniería por la importancia del vínculo académico-profesional en este Sello de Calidad Internacional y con CACEI y CIEES, la incorporación de evaluadores/as mexicanos/as al proyecto.

- Para las evaluaciones de programas españoles y mexicanos se aplica el mismo proceso y criterios de evaluación, con resultados similares en cuanto a la especialidad de ingeniería y ciclo formativo, y se promueve la participación de evaluadores/as de los dos países tanto en programas españoles y mexicanos, que cuentan con una formación anual idéntica.

- Entre el 2014-2020 ANECA ha otorgado 234 sellos distribuidos entre 35 instituciones de educación superior españolas y tres mexicanas, sin verse afectadas dichas evaluaciones por la pandemia, siguiendo las planificaciones establecidas al inicio del año 2020.

- De estos 234 sellos, 197 son de Grado y 66 de Máster. Todos los sellos mexicanos del nivel de Grado.

- Destacan los programas de ingeniería de la especialidad «Información y Comunicación», seguidas por «Industrial», «Química», «Civil/Construcción» y «Mecánica», que entre todas suman el $50 \%$ respecto al total de evaluaciones distribuidas entre las 21 especialidades establecidas por ENAEE.

- Al analizar en qué bloques de sub-resultados se han identificado más debilidades, «Proyectos de Ingeniería», «Aplicación práctica de la ingeniería» y «Comunicación y trabajo en equipo» son los que han alcanzado los datos más altos, tanto en los programas españoles como mexicanos.

- Desde el 2018 se realiza un metaevaluación anual del Programa de Sellos Internacionales de Calidad de ANECA a partir de las encuestas de satisfacción cumplimentadas por las universidades españolas y mexicanas y evaluadores/as que participan en cada convocatoria, en el que se gestionan las acreditaciones internacionales del Sello de ingeniería, de la que se han implantado 145 mejoras, siendo el $50 \%$ detectadas por el equipo SIC, entre las que destacan: una nueva estructura de presentación de las evidencias e informe de autoevaluación, generación de un documento de Preguntas Frecuentes y Glosario, redacción más detallada y exhaustiva en todos los informes de evaluación y un establecimiento de un plan de ahorro durante el proceso de evaluaciones. 
- Para atender a una de las sugerencias enviadas por las universidades, se realiza un análisis del número de créditos asignados efectivos a cada sub-resultado de aprendizaje a partir de los informes de evaluación con resultado positivo de la convocatoria 2018, con los siguientes resultados: en los programas de Grado un valor medio de asignación de 4 y en los de Máster de 3, aunque no se debe tomar como referencia estos números, porque para obtener el Sello lo importante no es la cantidad, sino la calidad de las actividades que están detrás de estos, para garantizar que todos los/as estudiantes trabajan cada sub-resultado, así como de la de los sistemas de evaluación con los que se cuentan para comprobar que todo el estudiantado los adquiere.

- En marzo del 2020, antes de realizarse la meta-evaluación anual prevista en el último semestre del año, ya se habían implantado dos nuevas mejoras: 1) la virtualización completa de las evaluaciones, debido a la pandemia provocada por la COVID-19, que al cierre del artículo se ha aplicado ya a 23 universidades españolas y 1 mexicana de forma satisfactoria y 2) los programas mexicanos que hayan obtenido la acreditación con CACEI en un plazo inferior a dos años, con resultado favorable en el momento de la evaluación del Sello, solo tendrán que someterse a la evaluación de los dos criterios específicos de este: Criterio 8: Resultados de Aprendizaje del Sello y el Criterio 9: Soporte institucional. Estas dos mejoras han incrementado el número de solicitudes mexicanas, recibidas en ANECA en junio de 2021, respecto a convocatorias anteriores.

\section{References}

ANECA: <www.aneca.es>

ANECA (2021a). Criterios de evaluación de los Sellos Internacionales de Calidad. Recuperado el 29 de julio de 2021 de, http://www.aneca.es/Programas-deevaluacion/Evaluacion-de-titulos/SIC

ANECA (2021b). Listado de títulos (programas formativos) universitarios con Sellos. Recuperado el 29 de julio de 2021 de, http://www.aneca.es/Programas-deevaluacion/Evaluacion-de-titulos/SIC/Resultados-de-los-Sellos-Internacionalesde-Calidad/Titulos-con-sello.

Asenjo y Asenjo (2020). Educación y europeísmo, un itinerario de encuentro transnacional. Revista Española de Educación Comparada, 36, 14-31. http:// revistas.uned.es/index.php/REEC/article/view/26145/21661

Bonilla-Calero y Serrano-García (2021). Auditorías virtuales en el Programa de Sellos Internacionales de Calidad (SIC) de ANECA en tiempos de pandemia , Revista Iberoamericana de Educación a Distancia, 24 (2), 245-261. https://doi. org/10.5944/ried.24.2.29092

Bonilla-Calero (2019). Los reconocimientos internacionales de calidad en ingeniería, informática y química por la Agencia Nacional de Evaluación de la Calidad y Acreditación (ANECA). Revista ACREDITAS por la Excelencia en Iberoamérica, 1, 10-15 . http://acreditas.com/images/acreditaso1.pdf_

CACEI: <http://cacei.org.mx/> 
CIEES: <https://www.ciees.edu.mx/>

Colomo y Esteban (2020). The European University: between Bologna and the Agenda 2020", 36, 54-73. Revista Española de Educación Comparada. http://revistas. uned.es/index.php/REEC/article/view/26179

ENAEE: <https://www.enaee.eu/>

ENAEE (2021). ENAEE Database of EUR-ACE Labelled Programmes. Recuperado el 29 de julio de 2021 de, https://eurace.enaee.eu/node/163

IIE: < https://www.iies.es/>

Kant (1999). La contienda entre las facultades de filosofía y teología. Madrid: Trotta. https://dialnet.unirioja.es/servlet/libro?codigo $=114903$

Washington Accord: <https://www.ieagreements.org/accords/washington/> 